Proc. Indian Acad. Sci. (Math. Sci.), Vol. 89, Number 1, January 1980, pp. 35-42. (C) Printed in India.

\title{
On duality in linear fractional programming
}

\author{
C R SESHAN \\ Department of Applied Mathematics, Indian Institute of Science, Bangalore 560012 \\ MS received 8 January 1979
}

\begin{abstract}
In this paper, a dual of a given linear fractional program is defined and the weak, direct and converse duality theorems are proved. Both the primal and the dual are linear fractional programs. This duality theory leads to necessary and sufficient conditions for the optimality of a given feasible solution. A unmerical example is presented to illustrate the theory in this connection. The equivalence of Charnes and Cooper dual and Dinkelbach's parametric dual of a linear fractional program is also established.
\end{abstract}

Keywords. Linear fractional programming; duality.

\section{Introduction}

In this paper a dual of a given linear fractional program is defined and this dual is also a linear fractional program. Kanti Swarup [11] has given a dual of a linear fractional program in which exists non-linearity in the constraints. Also, he did not prove the converse duality theorem. Kaska [7] has also given a dual of a linear fractional program which is constrained as the variable of the primal program. Chadha [2] has given a dual of a linear fractional program as a linear program which is nothing but the dual of the linear program obtained by Charnes and Cooper transformation of the original linear fractional program. Craven and Mond [4] have also given a dual of a linear fractional program such that both the primal and dual are linear fractional programs. Sharma and Swarup [10] have defined a dual of a linear fractional program in a different form but keeping the primal and dual as linear fractional programs.

Duals of nonlinear fractional programs have been proposed by Jagannathan [6], Bector [1], and Schaible [8,9]. The duals of Jagannathan and Schaible when applied to the linear fractional case give rise to the dual proposed by Chadha [2].

In the dual proposed by Sharma and Swarup [10], constant term does not appear in both the numerator and denominator of the objective function of the primal. This paper extends their definition to the general case where constant term is permitted to appear in the numerator and denominator of the objective function and the constraints of the dual are also generalised. This extension demands the revision of the proofs of the duality theorem. These proofs which make use of the results by Dinkelbach [5] are presented in this paper. This duality theory also 
leads to a set of necessary and sufficient conditions for a feasible solution to be optimal and these conditions are extensions of Kuhn-Tucker necessary optimality conditions.

In the last section, it is proved that Charnes and Cooper dual of a linear fractional program as a linear program can be obtained independently by making use of the results proved by Dinkelbach [5] where the given linear fractional programming problem is converted into a parametric linear programming problem.

\section{Dual of a linear fractional program}

Consider the primal linear fractional programming problem (P1)

Maximise $f(x)=\left(c^{2} x+a\right) /\left(d^{t} x+\beta\right)$

(P1) subject to $A x \leqslant b$

$$
x \geqslant 0 \text {. }
$$

where $A$ is an $(m \times n)$ matrix, $c, d, x$ are $(n \times 1)$ vectors, $b$ is an $(m \times 1)$ vector, $a, \beta$ are saalars and $t$ denotes transpose. Let

$$
S=\left\{x \in \dot{R}^{n} \mid A x \leqslant b, x \geqslant 0\right\} .
$$

Assume that $S$ is nonempty and bounded and that $f$ is not constant on $S$. Further assume that

$$
d^{t} x+\beta>0 \text { for every } x \text { in } R^{n}, x \geqslant 0 .
$$

This can be achieved if all the components of $d$ are nonnegative and $\beta>0$.

Deftue the dual (D1) corresponding to the primal (P1) as follows :

Minimise $g(u, v)=\left(c^{t} u+a\right) /\left(d^{t} u+\beta\right)$

subject to

(D1)

$$
\begin{aligned}
& c \cdot d^{t} u-d \cdot c^{t} u-A^{t} v \leqslant a d-\beta c \\
& a \cdot d^{t} u-\beta \cdot c^{t} u+b^{t} v \leqslant 0 \\
& u \geqslant 0, v \geqslant 0, u \in R^{n}, v \in R^{m} .
\end{aligned}
$$

\section{Theorem 1 (Weak duality)}

If $x$ is any feasible solution of $(\mathrm{Pl})$ and $(u, v)$ is any feasible solution of (D1), then

$$
f(x) \leqslant g(u, v) \text {. }
$$

Proof: Multiplying (2) by $x^{t}$

$$
c^{t} x \cdot d^{t} u-d^{t} x \cdot c^{t} u-x^{t} A^{t} v \leqslant a \cdot d^{t} x-\beta \cdot c^{t} x
$$

Multiplying (1) by $v^{*}$ and using (3)

$$
a \cdot d^{t} u-\beta \cdot c^{t} u \leqslant-b^{t} v \leqslant-x^{t} A^{t} v
$$

From (4) and (5)

$$
c^{t} x \cdot d^{t} u-d^{t} x \cdot c^{t} u+a \cdot d^{t} u-\beta \cdot c^{t} u \leqslant a \cdot d^{t} x-\beta \cdot c^{t} x
$$

i.e. $\quad\left(c^{t} x+a\right)\left(d^{t} u+\beta\right) \leqslant\left(c^{t} u+a\right)\left(d^{t} x+\beta\right)$. 
Hence,

$$
f(x)=\frac{c^{t} x+a}{d^{t} x+\beta} \leqslant \frac{c^{t} u+a}{d^{t} u+\beta}=g(u, v) .
$$

Corollary 1: If $x$ is any feasible solution of (P1) and $(u, v)$ is any feasible solution of (D1) such that $f(x)=g(u, v)$, then $x$ solves (P1) and $(u, v)$ solves (D1).

Proof is obvious.

\section{Theorem 2 (Direct duality)}

If $\bar{x}$ solves (P1), then there exists $(\bar{u}, \bar{v})$ which solves (D1) such that $f(\tilde{x})=g(\bar{u}, \bar{v})$.

Proof. Let $\lambda=\left(c^{t} \bar{x}+a\right) /\left(d^{t} \bar{x}+\beta\right)$.

Consider the linear programming problem (P2).

Maximise $\left(c^{t} x+a\right)-\lambda\left(d^{t} x+\beta\right)$

(P2) subject to $A x \leqslant b, x \geqslant 0$.

Dinkelbach [5] has proved that $\bar{x}$ also solves (P2) and the optimal value of the objective function in $(\mathrm{P} 2)$ is 0 .

Consider the dual of (P2) and denote it by (D2)

Minimise $b^{\boldsymbol{t}} v+a-\lambda_{\beta}$

(D2) subject to $A^{t} v \geqslant c-\lambda d$

$$
v \geqslant 0, v \in R^{m}
$$

Let $\bar{v}_{1}$ solve (D2). By duality theorem of linear programming

$$
b^{*} \bar{v}_{1}+a-\lambda \beta=0 \text {. }
$$

Let $\quad \bar{u}=\bar{x}$ and $\bar{v}=\bar{v}_{1}\left(d^{t} \bar{x}+\beta\right)$

$$
\begin{aligned}
c \cdot d^{t} \bar{u}-d \cdot & c^{t} \bar{u}-A^{t} \bar{v} \leqslant c \cdot d^{t} \bar{x}-d \cdot c^{t} \bar{x}-(c-\lambda d)\left(d^{t} \bar{x}+\beta\right) \\
& =c \cdot d^{t} \bar{x}-d \cdot c^{t} \bar{x}-c\left(d^{t} \bar{x}+\beta\right)+d\left(c^{t} \bar{x}+a\right) \\
& =a d-\beta c .
\end{aligned}
$$

Multiplying (8) by $d^{t} \bar{x}+\beta$,

$$
b^{t} \bar{v}+a\left(d^{t} \bar{x}+\beta\right)-\beta\left(c^{t} \bar{x}+a\right)=b^{t} \bar{v}+a \cdot d^{t} \bar{u}-\beta \cdot c^{t} \bar{u}=0 .
$$

Hence $(\bar{u}, \bar{v})$ is a feasible solution of (D1).

$$
f(\bar{x})=\frac{c^{t} \tilde{x}+a}{d^{t} \bar{x}+\beta}=\frac{c^{t} \bar{u}+a}{d^{t} \bar{u}+\beta}=g(\bar{u}, \bar{v}),
$$

$(\bar{u}, \bar{v})$ solves (D1), because of corollary.

Theorem 3 (converse duality)

If $(\bar{u}, \bar{v})$ solves (D1) then there exists an $\bar{x}$ which solves (P1) such that $f(\bar{x})=$ $g(\bar{u}, \bar{v})$.

Proof: Let $\lambda=\left(c^{t} \bar{u}+a\right) /\left(d^{*} \bar{u}+\beta\right)$. 
Consider the linear programming problem (P3)

Minimise $\left(c^{t} u+a\right)-\lambda\left(d^{t} u+\beta\right)$

(P3) subject to $c \cdot d^{t} u-d \cdot c^{t} u-A^{t} v \leqslant \alpha d-\beta c$

$$
\begin{aligned}
& a \cdot d^{t} u-\beta \cdot c^{t} u+b^{t} v \leqslant 0 \\
& u \geqslant 0, v \geqslant 0 .
\end{aligned}
$$

Dinkelbach [S] has proved that $(\bar{u}, \bar{v})$ also solves (P3) and the optimal value of the objective function in (P3) is 0 .

Consider the dital of (P3) and denote it by (D3)

Maximise $(-a d+\beta c)^{t} y+a-\lambda \beta$

subject to

$$
\begin{aligned}
& \text { (D3) }\left(-c \cdot d^{t}+d \cdot c^{t}\right)^{t} y+(-a d+\beta c) \mu \leqslant c-\lambda d \\
& A y-b \mu \leqslant 0 \\
& y \geqslant 0, \mu \geqslant 0, y \in R^{n}, \mu \in R .
\end{aligned}
$$

Let $(\bar{y}, \bar{\mu})$ solve $(\mathrm{D} 3)$.

By the duality theorem of linear programming

$$
-a d^{\prime} \bar{y}+\beta \cdot c^{*} \bar{y}+a-\lambda \beta=0
$$

$\bar{\mu} \neq 0$. For if $\overline{\boldsymbol{u}}=0$, from (10) we get $A \bar{y} \leqslant 0, \bar{y} \geqslant 0$. Since $S$ is nonempty, there exists an $x$ on $S$ such that $A x \leqslant b, x \geqslant 0$. Then $A(x+t \bar{y}) \leqslant b, x+t \bar{y} \geqslant 0$ for every $t>0$. Hence $x+t \bar{y}$ is in $S$ for every $t>0$ which is a contradiction if $\bar{y} \neq 0$ since $S$ is bounded.

If both $\bar{\mu}=0$ and $\bar{y}=0$, then from (11), $a-\lambda \beta=0$. From (9), we get $c \geqslant \lambda d$. Let $x$ be any feasible solution of (P1). We have $c^{t} x \geqslant \lambda d^{t} x$ and $a=\lambda \beta$. Hence $\left(c^{*} x+a\right) \geqslant \lambda\left(d^{t} x+\beta\right)$, i.e., $f(x) \geqslant \lambda$. But by Theorem $1, f(x) \leqslant g(\bar{u}, \bar{v})=\lambda$. Therefore $f(x)=\lambda$ for every feasible solution $x$ of (P1) which implies that $f$ is a constant on $S$, a contradiction to our assumption.

Therefore $\bar{\mu}>0$. Let $\bar{x}=\bar{y} / \bar{\mu}$. From (10), $A \bar{x} \leqslant b$. Also $\bar{x} \geqslant 0$. Hence $\bar{x}$ is a feasible solution of (P1).

Applying complementary slackness theorem of linear programming to (P3) and (D3) we get

$$
\begin{aligned}
& c^{t} \bar{y} d^{t} \bar{u}-d^{t} \bar{y} c^{t} \bar{u}-\bar{y}^{t} A^{t} \bar{v}-a \cdot d^{t} \bar{y}+\beta \cdot c^{t} \bar{y}=0 \\
& a \bar{\mu} d^{t} \bar{u}-\beta \bar{\mu} c^{t} \bar{u}+\bar{\mu} b^{t} \bar{v}=0 \\
& -d^{t} \bar{u} c^{t} \bar{y}+c^{t} \bar{u} d^{t} \bar{y}-a \bar{\mu} d^{t} \bar{u}+\beta \bar{\mu} c^{t} \bar{u}-c^{t} \bar{u}+\lambda d^{t} \bar{u}=0 \\
& \bar{y}^{t} A^{t} \bar{v}-\bar{\mu} b^{t} \bar{v}=0 .
\end{aligned}
$$

Adding (12), (13) and (15)

$$
\begin{aligned}
& c^{t} \bar{y} d^{t} \bar{u}-d^{t} \bar{y} c^{t} \tilde{u}-a d^{t} \bar{y}+\beta c^{t} \bar{y}+a \bar{\mu} d^{t} \tilde{u}-\beta \bar{\mu} c^{t} \bar{u}=0 \\
& \left(c^{t} \bar{y}+a \bar{\mu}\right)\left(d^{t} \bar{u}+\beta\right)=\left(d^{t} \bar{y}+\beta \bar{\mu}\right)\left(c^{t} \bar{u}+a\right) .
\end{aligned}
$$


Hence

$$
\frac{e^{t} \bar{y}+a \bar{u}}{d^{t} \bar{y}+\bar{\beta}_{\bar{\mu}}}=\frac{c^{t} \bar{u}+\alpha}{d^{t} \bar{u}+\beta} .
$$

Therefore

$$
f(x)=\frac{c^{t} \bar{x}+a}{d^{t} \bar{x}+\beta}=\frac{c^{t} \tilde{u}+a}{d^{t} \bar{u}+\beta}=g(\tilde{u}, \bar{v}) .
$$

Hence $\bar{x}$ solves (P1) because of the corollary.

Note 1 : Converse duality theorem can be deduced directly from direct duality theorem. This can be seen by the following argument. $(\bar{u}, \bar{v})$ solves (D1). Since $S$ is compact, (P1) has a finite optimal solution say $\bar{x}$. By direct duality theorem, there exists $\left(\bar{u}_{1}, \bar{v}_{1}\right)$ which solves (D1) such that $f(\bar{x})=g\left(\bar{u}_{1}, \bar{v}_{1}\right)$. Hence $f(x)=$ $g\left(\bar{u}_{1}, \bar{v}_{1}\right)=g(\bar{u}, \bar{v})$.

Note 2: We have not used the assumption that $S$ is bounded in proving either the weak duality theorem or direct duality theorem. Even in the case of converse duality theorem, we can replace that assumption by the following weaker assumption

$$
A y \leqslant 0, \quad y \geqslant 0 \text { implies } y=0 \text {. }
$$

\section{Some remarks}

Remark 1: The problem (P1) is equivalent to the problem (Q1)

Maximise $f(x)=\left(c^{t} x+a x_{n+1}\right) /\left(d^{t} x+\beta x_{n+1}\right)$

subject to $A x \leqslant b$

$$
\begin{gathered}
x_{n+1} \leqslant 1 \\
-x_{n+1} \leqslant-1 \\
x \geqslant 0, x_{n+1} \geqslant 0, x \in R^{n}, x_{n+1} \in R .
\end{gathered}
$$

In this form the problem (P1) is in the same form as the (LFP) considered by Sharma and Swarup [10]. We can write the dual (E1) of (Q1) as per definition of dual by Sharma and Swarup as follows:

Minimise $\left(c^{\imath} u+a u_{n+1}\right) /\left(d^{*} u+\beta u_{n+1}\right)$

subject to

$$
\begin{aligned}
& c \cdot d^{t} u-d \cdot c^{\prime} u-A^{z} v \leqslant(a d-\beta c) u_{n+1} \\
& a \cdot d^{z} z-\beta \cdot c^{t} u-v_{m+1}+v m \leqslant 0 \\
& \quad b^{t} v+v_{m+1}-v_{m+2} \leqslant 0 \\
& u, v, u_{n+1}, v_{m+1}, v_{m+2} \geqslant 0, u \in R^{n}, v \in R_{m}, u_{n+1}, v_{m+1}, v_{m+2} \in R .
\end{aligned}
$$

Any feasible solution $(u, v)$ of $(\mathrm{D} 1)$ gives rise to many feasible solutions $\left(u^{\prime}, v^{\prime}\right.$, $\left.u_{n+1}^{\prime}, v_{m+1}^{\prime}, v_{m+2}^{\prime}\right)$ of (E1) with $u_{n+1}^{\prime}=a$, where $a>0$ is any real number, 
$u^{\prime}=a \cdot u, v=a \cdot v$, and $v_{m+1}, v_{m+2}$ are chosen suitably. Also the corresponding objective function values become all equal. Conversely any feasible solution $\left(u^{\prime}, v^{\prime}\right.$, $\left.u_{n+1}^{\prime}, v_{m+1}^{\prime}, v_{m+2}^{\prime}\right)$ of (E1) with $u_{n+1}^{\prime} \neq 0$ gives rise to a feasible solution $(u, y)$ of (D1) where $u=u^{\prime} / u_{n+1}^{\prime}, v=v^{\prime} / u_{n+1}^{\prime}$ with the same objective function value. Feasible solutions of (E1) with $u_{n+1}^{\prime}=0$ do not correspond to any feasible solution of (D1). Therefore our dual (D1) is not equivalent to dual (E1). There is a one to many correspondence between feasible solutions of (D1) and a subset of feasible solutions of (E1). Thus (D1) is a much simpler dual than (E1) for the problem (P1).

Remark 2. The dual (R1) of the dual (D1) is

Maximise $\left(c^{t} x+a\right) /\left(d^{t} x+\beta\right)$

subject to

$$
\begin{aligned}
& c \cdot d^{t} z-c \cdot d^{t} x-d \cdot c^{t} z+d \cdot c^{t} x \leqslant(a d-\beta c)(\lambda-1) \\
& a \cdot d^{t} z-a \cdot d^{t} x-\beta \cdot c^{t} z+\beta \cdot c^{\prime} x \leqslant 0 \\
& A z-\lambda b \leqslant 0 \\
& x, z, \lambda \geqslant 0, x, z \in R^{n}, \lambda \in R .
\end{aligned}
$$

Any feasible solution $x$ of (Pl) gives rise to a feasible solution of (R1) if we take $z=x$ and $\lambda:=1$. Further the objective function values are equal. But the converse is not true. Hence dual of (DI) is not equivalent to (P1).

Remark 3. The above duality theory leads to necessary and sufficient conditions for a feasible solution $x$ of the primal to be optimal. From the proof of direct duality theorem and weak duality theorem, it is easy to see that a feasible solution $x$ of (P1) is an optimal solution of (P1) if and only if there exists a $v \geqslant 0, v \in R^{m}$ such that

$$
\begin{aligned}
& c \cdot d^{t} x-d \cdot c^{t} x-A^{t} v \leqslant a d-\beta c \\
& a \cdot d^{t} x-\beta \cdot c^{t} x+b^{t} v \leqslant 0 .
\end{aligned}
$$

Of the above two conditions, condition (23) is the Kuhn-Tucker necessary optimality conditians for (P1).

\section{Numerical example}

\section{Primal problem}

$$
\begin{array}{ll}
\text { Maximise }\left(3 x_{1}+3 x_{2}+2 x_{3}+1\right) /\left(2 x_{1}+x_{2}+x_{3}+1\right) \\
\text { subject to } & 2 x_{1}+5 x_{2}+x_{3} \leqslant 2 \\
& x_{1}+2 x_{2}+3 x_{3} \leqslant 3 \\
& x_{1}, x_{2}, x_{3} \geqslant 0 .
\end{array}
$$

Solving, we get that $x_{1}=0, x_{2}=3 / 13, x_{3}=11 / 13$ is an optimal solution and the optimal value of the objective function is $44 / 27$. 


\section{Dual problem}

$$
\text { Minimise }\left(3 u_{1}+3 u_{2}+2 u_{3}+1\right) /\left(2 u_{1}+u_{2}+u_{3}+1\right)
$$

subject to

$$
\begin{aligned}
& -3 u_{2}-u_{3}-2 v_{1}-v_{2} \leqslant-1 \\
& 3 u_{1}+u_{3}-5 v_{1}-2 v_{2} \leqslant-2 \\
& u_{1}-u_{2}-v_{1}-3 v_{2} \leqslant-1 \\
& -u_{1}-2 u_{2}-u_{3}+2 v_{1}+3 v_{2} \leqslant 0 \\
& u_{1}, u_{2}, u_{3}, v_{1}, v_{2} \geqslant 0 .
\end{aligned}
$$

Solving this we get that $u_{1}=0, u_{2}=3 / 13, u_{3}=11 / 13, v_{1}=7 / 13, v_{2}=1 / 13$ is an optimal solution and the optimal value of the objective function is $44 / 27$. Other optimal solutions are:

$$
\begin{aligned}
& u_{1}=0, u_{2}=0, u_{3}=17 / 10, v_{1}=7 / 10, v_{2}=1 / 10 \text { and } \\
& u_{1}=0, \quad u_{2}=17 / 37, \quad u_{3}=0, \quad v_{1}=14 / 37, \quad v_{2}=2 / 37 .
\end{aligned}
$$

These results are as per the expertations of theorems 2 and 3 .

\section{A note on Charnes and Cooper dual of a linear fractional program}

Ciarnes and Cooper (1962) converted the linear fractional programming problem (P1)

$$
\text { Maximise }\left(c^{t} x+a\right) /\left(d^{t} x+\beta\right)
$$

(P1) subject to $A x \leqslant b, x \geqslant 0$

under the assumption $d^{t} x+\beta>0$ for every feasible $x$, into the linear programming problem (P4)

Maximise $c^{\star} y+a t$

(P4) subject to $A y-b t \leqslant 0$

$$
\begin{aligned}
& d^{t} y+\beta t=1 \\
& y \geqslant 0, t \geqslant 0
\end{aligned}
$$

using the transformation

$$
t=1 /\left(d^{t} x+\beta\right), y=x t
$$

The Charnes and Cooper dual of (PI) is given by (D4)

\section{Minimise $\lambda$}

(D4) subject to $A^{\prime} u+\lambda d \geqslant c$

$$
\begin{aligned}
& -b^{t} u+\lambda \beta \geqslant a \\
& u>0, \lambda \text { unrestricted }
\end{aligned}
$$


Dinkelbach (1967) converted (PI) into the parametric linear programming problem $\left(P_{\lambda}\right)$

$$
\text { Maximise }\left(c^{t} x+a\right)-\lambda\left(d^{t} x+\beta\right)
$$

$\left(P_{\lambda}\right)$ subject to $A x \leqslant b, x \geqslant 0$.

He proved that $\bar{x}$ solves $(\mathrm{Pl})$ and $\lambda$ is the optimal value of the objective function in (P1) if and only if $\bar{x}$ solves $\left(P_{\lambda}\right)$ and $F(\lambda)=0$, where $F(\lambda)$ is the maximum of the objective function of $\left(P_{\lambda}\right)$. He also proved that $F(\lambda)$ is a monotonic decreasing function of $\lambda$. Therefore the problem (P1) can be viewed as the one in which we have to find a $\lambda$ such that $F(\lambda)=0$ (i.e., to minimise $\lambda$ such that $F(\lambda) \leqslant 0$ ). Considering the dual of $\left(P_{\lambda}\right)$ we get

$$
F(\lambda)=\min \left\{b^{t} u+a-\lambda \beta: A^{t} u \geqslant c-\lambda d, u \geqslant 0\right\} .
$$

Hence the dual of the linear fractional programming problem (P1) is

Minimise $\lambda$

$$
\begin{aligned}
\text { subject to } & A^{t} u \geqslant c-\lambda d \\
& b^{t} u+a-\lambda \beta \leqslant 0 . \\
& u \geqslant 0, \lambda \text { unrestricted. }
\end{aligned}
$$

Hènce Charnes and Cooper dual of a linear frastional progra'n and Dinkelbach's parametric dual of a linear fractional program are essentially the same.

\section{Acknowledgement}

The author wishes to express his gratitude to Dr V G Tikekar, for his encouragement, guidance and for the fruitful diszussions that the author had with him.

\section{References}

[1] Bector C R 1973 Z. Opns. Res. 17183

[2] Chadha S S 1971 Z. Angew. Math. Mech. 51560

[3] Charnes A and Cooper W W 1962 Nav. Res. Log. Q. 9, 181

[4] Craven B D and Mond B 1973 J. Math. Anal. Appl. 42507

[5] Dinkelbach W 1967 Manage. Sci. 13492

[6] Jagannathan R $1973 Z$. Opns. Res. 171

[7] Kaska J 1969 Econ. Mat. Obzor. 5442

[8] Schajble S 1974 Z. Opns. Res. 17187

[9] Schaible S 1976 Manage. Sci. 22858

[10] Sharma I C and Swarup K 1972 Z. Opns. Res. 1691

[11] Swarup K 1968 Untermehmensforchung 12106 